\section{Yashraj Jain}

is research executive at the

Direct Marketing Association

(DMA) (UK). He is responsible for revamping the DMA's

research programme, helping to develop pioneering insight based around ten research hubs.

Outputs from the research have been widely disseminated in partnership with major clients and through marketing media. He is also part-time research administrator at the career development centre of the University of Westminster.
Keywords: direct marketing, direct mail, email marketing, social media, consumer attitudes, DMA

\section{Understanding consumer channel preferences}

Yashraj Jain

Direct Marketing Association (UK), DMA House, 70 Margaret Street, London, W1W 8SS, UK Tel: +44 (0)20 72913359 Fax: +44 (0)20 72913301 E-mail: Yashraj.Jain@dma.org.uk

\section{Business Intelligence}

\section{DMA print tracking: Attributes of media channels}

\author{
Yashraj Jain and David Reed \\ Received (in revised form): 31st July 2013
}

\begin{abstract}
Marketers have to make choices about the media channels they use to distribute their message. This research study examines the attributes that consumers ascribe to different channels and how important these are when receiving marketing information. It discovers significant strengths for direct mail in 8 out of 11 of the attributes considered.

Journal of Direct, Data and Digital Marketing Practice (2013) 15, 56-63. doi:10.1057/dddmp.2013.35
\end{abstract}

\section{Background}

Since 2008, the Direct Marketing Association (DMA) (UK) has been commissioning a series of research reports, in association with its insight partner fast.MAP. These have focused on subjects of interest to direct and digital marketers, such as the Financial services tracking report, which measures levels of trust and intention to purchase a range of financial products; the Data tracking report, which monitors attitudes towards the collection and usage of personal information by marketers; and the Email tracking report, which assesses how consumers interact with emails from brands.

In 2013, the DMA commissioned a new report, From letterbox to inbox, sponsored by HP, to look at consumer attitudes towards key media channels (direct mail, email, door drops, inserts, phone calls, SMS, social network posts). The focus of the research was to understand underlying preferences towards each of these and to what extent consumers anticipate the migration of communications online.

This paper examines specific findings from the report, highlighting the attributes that consumers identify within marketing channels and how important these attributes are when consumers consider marketing information. This print tracking report from the DMA randomly selected 1,232 individuals from the fast.MAP Consumer Voice panel to provide a representative sample of the UK population. They were given an online, self-completion questionnaire of 28 questions covering actual receipt of marketing, preferences by channel, treatment and actions with items received, and views about the changing media landscape. 


\section{Attributes may affect attitudes}

\section{Calculating preference through importance of attributes}

\section{Introduction}

When marketers develop campaigns to promote a product or service, the focus is usually on how to leverage brand attributes and brand equity in order to convince individuals to make a purchase. The strength of these brand qualities varies tremendously and may require reinforcement through promotional and pricing mechanisms.

What is less often considered is the extent to which the medium through which these campaigns are to be delivered will itself have a bearing on how persuasive a communication is likely to be. Consumers may already hold a belief about the brand being marketed, but they may also have beliefs about specific attributes within the channels through which they receive information about that brand. The importance of these media attributes may have an effect on the attitude formed by a consumer as a result of any communication, just as much as an existing perception of the brand may make them more or less receptive to the marketing message.

\section{Application of Fishbein model}

Fishbein has become a standard method for developing a single score for the beliefs and attitudes that consumers have towards brands. The model is used by the DMA (UK) to assess how likely it is that a consumer's behaviour will change as a result of these attitudes, so that a marketer can understand whether purchasing will ultimately be influenced.

Using this model, often marketers derive a score to determine the overall attitude consumers have about a particular brand as against its competitors.

First, customers are asked to rate the importance of the different characteristics of a product (or a service). For example, if a customer believes that smell is an extremely important characteristic when deciding which coffee to purchase, they may give it a score of 9 out of 10 on the importance scale. On the other hand, the packaging of the coffee may not be that important, scoring 4 out of 10 . Next, customers are asked to rate different brands on how they fare for each of the characteristics. In our example, say Brand A scored 6 for smell but -8 for packaging and Brand $\mathrm{B}$ got 10 for smell and -1 for packaging (on a scale ranging from -10 to +10 ).

We can then derive the Fishbein score for each brand by multiplying the scores for the two variables:

Brand score $=[$ Importance of smell $\times$ Rating for smell $]+[$ Importance of packaging $\times$ Rating for packaging]

In our example, this produces the following scores:

Brand $A=[9 \times 6]+[4 \times-8]=54-32=22$

Brand $\mathrm{B}=[9 \times 10]+[4 \times-1]=90-4=86$

Thus, Brand B would be the preferred choice.

A modified version of this model was applied where the characteristics - or attributes as the DMA puts it - of marketing channels were rated on an importance scale and UK consumers were then asked to select which one marketing channel best reflected these attributes according to them. Consumers were not asked to score the different marketing channels individually for each characteristic but to select the one they felt best 


\section{Establishing target attributes for research}

demonstrated it. This meant that no overall score as such was calculated. This was mainly due to logistical reasons to keep the survey length manageable, while still in essence deriving some understanding of which marketing channels fare better than their 'competitors' on different attributes.

As M. Fishbein and I. Ajzen wrote in Belief, Attitude, Intention, and Behaviour: An Introduction to Theory and Research (Addison-Wesley, 1975), 'The purpose of any persuasive communication is to produce change in some dependent variable, whether a belief, an attitude, an intention, or a behaviour ... The assumption is that acceptance of the source belief statements contained in the message will lead to a change in the dependent variable. It may thus be argued that a message is effective to the extent that the receivers accept the source beliefs it contains'.

If those source beliefs are considered to reside within marketing channels, rather than the brand using them, then this model makes it possible to identify how consumers rate different attributes within media and which channels possess those important attributes. The insight gained from this study can then allow marketers to tap into those attributes of a marketing channel for which consumers have a high regard.

\section{Methodology}

To explore this, a number of questions were constructed for the print tracking survey based on a modified version of the Fishbein model. This provides a multi-attribute model in which overall attitudes can be assessed as a reflection of the strength of belief about a particular attribute combined with the consumer's evaluation of whether that attribute is good or bad.

For the purposes of this research, a list of attributes was developed. This was possible by looking at past market research surveys carried out by the DMA to find out what basic characteristics marketing channels have. The list of attributes was put forward for discussion in a focus group that consisted of research suppliers and experts from the print industry. When drafting a list, it was important to remember that this was a consumer survey, and therefore the terms used were simple and easy to relate to. Moreover, it was hoped that the implications of the findings would be of some practical use to marketers. Finally, the following list was drawn up that reflects dimensions of benefit to marketers and over which they have a degree of control. These were:

- Tangible

- Trustworthy

- Authoritative

- Appearance

- Memorable

- Personalization

- Relevance

- Attention-grabbing

- Content

- Ability to share with others

- Easy to respond to 
This list is not exhaustive and over time will evolve. For example, it could be argued that content and relevance are interchangeable or what the meaning of relevance is - relevant timing of receiving marketing communication or relevant to the consumer's needs. Future research on the subject could explore this further and refine the list.

Survey respondents were then asked the following questions:

- Which marketing channel do you feel best reflects these attributes?

- Now thinking about marketing information in general, how important are the following attributes to you in terms of receiving marketing information?

Results from these questions are considered below.

\section{Research findings}

\section{Functional and emotional attributes}

\section{Which marketing channel best reflects each attribute?}

In recognizing certain attributes within each marketing channel, consumers are to some extent simply identifying the inherent or functional characteristics of that channel. Thus, direct mail scores highest on being tangible as might be expected when it is considered against digital channels such as email, SMS and social media or a phone call. Similarly, email gains the second highest attribute score on being easy to respond to, a feature that is built in to this particular channel (Figure 1).

Evidence that something more is happening can be found in the scores for this attribute achieved by door drops (10 per cent) and inserts ( 3 per cent), which are substantially lower, even though these channels are also physical in nature. It may be that the time that consumers spend in handling a direct mail pack - through opening it, reading its contents and often retaining it - reinforces its tangible nature more strongly than items that are more rapidly considered and dealt with.

This is further reinforced when the attribute for ability to share with others is considered. Email scores highest (45 per cent), which might be anticipated given the ease with which a message can be forwarded or shared using

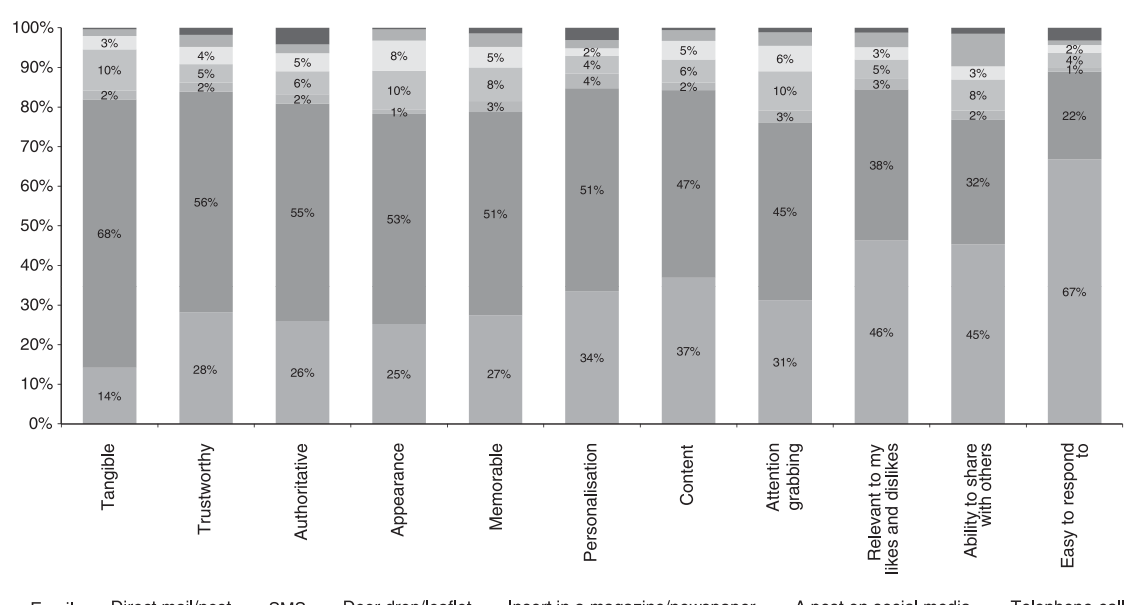

Figure 1: Attributes by Marketing Channel 


\section{Perception of source affects receptiveness}

built-in social tools. Yet direct mail is rated second (32 per cent) while social media are only scored at 8 per cent for this quality. It can hardly be argued that sharing a mail pack is easier than sharing content in social media. Therefore, it seems to be the case that once a medium has established a certain standing among consumers, it benefits from a halo effect across other attributes where it might be expected to rank lower.

Direct mail demonstrates this by scoring higher than all other media on 8 out of 11 attributes considered. In addition to being tangible, three of these attributes can be directly influenced by marketers - appearance, personalization and content. It may also be argued that two further attributes - memorable and attention-grabbing — are the consequence of what marketers do with design, copy and data variables. By contrast, email appears to be succeeding in establishing its functional attributes in the mind of the consumer - easy to respond to, relevant, ability to share with others - yet scores considerably lower on the other attributes, even where these are equally under the control of marketers as direct mail.

Where this extended attribute benefit really comes into play is in more emotional dimensions. When asked about channels demonstrating trustworthiness and being authoritative, direct mail attracts twice the level of scores given to email, with all other channels having very low scores. Marketers who need to communicate these qualities in particular will do well to consider the weight that direct mail carries with consumers.

\section{How important are attributes when receiving marketing information?}

Fishbein has demonstrated that receptiveness to marketing messages is affected by the consumer's perception of the source beliefs of the communicator. That is, if the individual believes the source of a message, they are more likely to believe the message itself and be influenced by its content. When it comes to the channels marketers use, source beliefs can be understood as the attributes ascribed to a channel. The more strongly those attributes are identified with a channel, the more receptive the consumer will be to messages received that way (Figure 2).

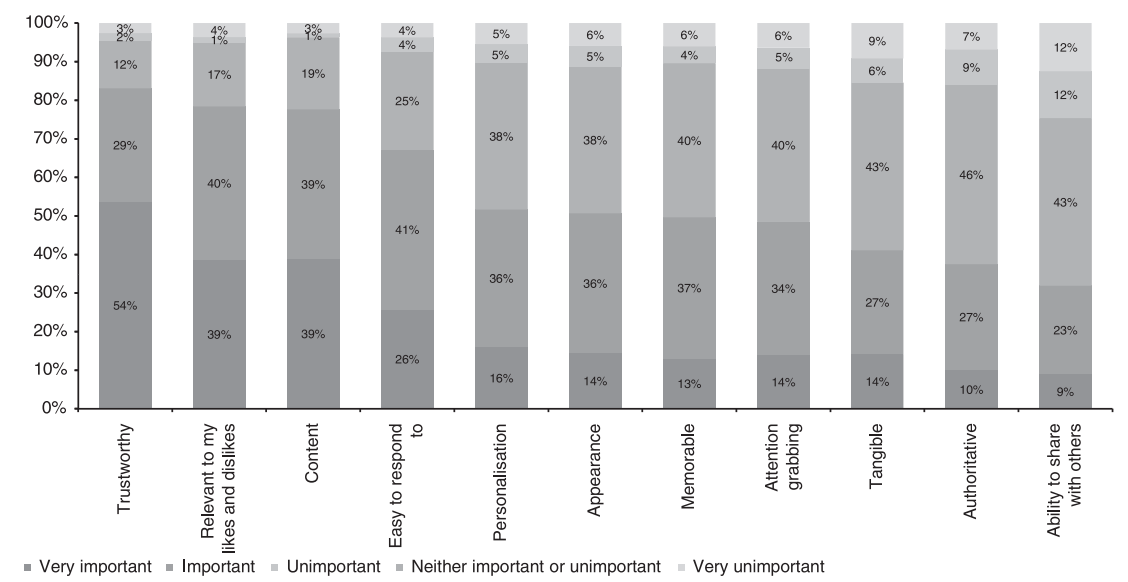

Figure 2: Importance of Attributes in Marketing Information 


\section{Hygiene factors and neutral factors}

\section{ExactTarget survey of consumer channel use}

\author{
Six factors determine \\ choice
}

Attributes have different levels of importance when receiving marketing information. Trustworthy is the most highly rated quality, with 83 per cent of consumers stating that it is important or very important. That a majority of consumers (54 per cent) rate it as very important underlines the strength of this attribute, since achieving the highest ranking is difficult and this is the only one to be thus rated by more than half of survey respondents.

Efforts made by marketers to ensure that the information they send is of interest and engages the consumer are clearly merited, since relevance and content attract the second and third highest levels of importance at 79 and 78 per cent important or very important, respectively. One other attribute - easy to respond to - is also rated highly by a strong majority of consumers.

Four other attributes in the receipt of marketing information can be thought of as hygiene factors since they rate as important or very important to just half of consumers. These are personalization, appearance, memorable and attention-grabbing. But for the remaining three attributes considered - tangible, authoritative and ability to share with others more consumers are neutral than see them as of importance. This means these attributes are unlikely to influence receptiveness to marketing messages. Given the attention that digital marketers have recently been paying to the incorporation of social tools into all channels, the indifference consumers express towards the ability to share with others suggests that this technique should not be solely relied on to drive marketing performance.

\section{Comparison with other channel studies}

Attributes of channels used by marketers reflect both innate qualities of those channels, such as their ability to be personalized or shared, and emotional or soft dimensions, such as being trustworthy or memorable. These attributes can be defined in a number of different ways and selected according to the needs of a given research project.

To understand the insights gained from the DMA print tracking report, it is useful to compare its attributes list with that used in another survey.

Since 2008, ExactTarget has carried out three studies into consumers' use of different channels for interpersonal communication and attitudes towards marketing communications. For its 2012 survey, it collected 1,481 responses via the MarketTools TrueSample panel, which is representative of the US population.

ExactTarget considered how consumers determine the proper channel for their communication needs and identified six factors.

Content: The study has identified that consumers are now more selective about the channels through which they will allow marketing messages, even if they are fans of a brand. Marketing messages are not appropriate in every context - something marketers need to understand when drawing up their media plan.

Immediacy: When contacting their own family and friends, immediacy is critical and draws the user to social networks, instant messaging, phone and SMS. For marketing messages that are genuinely urgent, these 
channels will perform well, but if there is less of a time factor, email or direct mail may be more positively perceived. (This may help to explain the very low scores seen for these immediate channels in the DMA survey - consumers may be applying a filter that does not acknowledge that marketing messages require the speed at which these channels operate.)

Accessibility: One of the effects identified by the DMA in its study is the 'mantelpiece effect'. This is where consumers keep items of direct mail to act on or share later. In its survey, the DMA found that consumers tend to decide more quickly on email messages, but rarely kept them for later. In the ExactTarget study, the need to reference a message later is identified as a positive aspect of email, since it can be accessed from multiple devices (smartphone, tablet, computer) wherever the individual may be. This attribute is clearly worth considering when deciding between possible marketing channels.

Privacy: Consumers appear to be building a set of preferences that reflects the track record of different channels in protecting their conversations. This places more confidence in text messaging, telephone and email, according to ExactTarget, than in social channels (although this does not always prevent consumers from using those options to make private statements). This attribute was not considered in the DMA survey as it was not looking at messages generated by consumers themselves, only those originated by marketers. However, the DMA print tracking report did look at trustworthiness - findings from the DMA Data tracking report suggest a close link between positive privacy controls and trust.

Formality: A degree of etiquette can still be found among consumers, even with the increase in social conversation. ExactTarget found that as a result, they still retain a view of marketing messages as formal communications, which leads to a preference for more formal channels when receiving them. This could prove to have a significant impact on the ability of consumers to receive the content of a message if the channel through which it arrives validates their perception of the source beliefs of the sender - that is, a brand is serious about what it is saying if it uses formal channels for its messages.

Initiation: ExactTarget identified this attribute as the most important for consumers. The channel they use to start a conversation with a brand is then considered appropriate for a marketing response. But care needs to be taken not to continue a private conversation with the consumer (eg in response to a tweet about poor customer service) in a public channel, but to switch into a more confidential mode in that channel if one is available (such as a direct Twitter message).

\section{Conclusions}

As a result of the DMA findings, marketers need to be aware that consumers ascribe specific attributes to marketing channels that are independent of the messages being sent through them. These attributes preexist and work for or against the ability of the consumer to receive the content of a message and be influenced by it. 
These scores and ratings are what consumers inherently feel about these attributes, and which source beliefs they regard as important and which they do not. Marketers should therefore aim to tailor their campaigns to leverage consumers' perceptions of these channels. The findings also help marketers determine the key points in the customer journey where different channels should be used.

The findings clearly show that direct mail and email lead the pack as primary channels and the remaining act as catalysts to augment the effect of these two. Similarly, as direct mail scores high on trust, personalization and content, it could be that brands should use this medium in the initial stages of their campaigns to build or retain trust, then go on to drive customers online and introduce email to engage and target their customers, since email scores high on relevancy and being easy to respond to.

The youngest channels available - social networks, SMS - have yet to establish any significant standing on these core attributes, which suggests that care should be taken about using them for communication with customers, as opposed to prospects. (Indeed, the DMA study found a real potential for brand damage if companies migrate all of their communications online without supporting the option to receive them in print.)

Consumers also have a clear hierarchy of importance for these attributes. Features that have recently excited marketers, such as the ability to share content using social tools, are not highly thought of by most individuals. The effectiveness of a message can therefore be improved if the right channels are selected and the design and format of the message optimized to leverage the inherent attributes found in those channels. 